\title{
Combining of Local Histogram and Local Slice Feature Vectors for Classifying Diffuse Lung Opacities in Thin-Section Computed Tomography Images
}

\author{
Yoshihiro Mitani, Yusuke Fujita, Naofumi Matsunaga, and Yoshihiko Hamamoto
}

\begin{abstract}
The classification of diffuse lung opacities in thin-section computed tomography (HRCT) images is an important step for developing a computer-aided diagnosis (CAD) system. In designing the CAD system for classifying diffuse lung opacities in HRCT images, a local histogram feature vector approach has been proposed and shown to be effective. Furthermore, a local slice feature vector approach unlike a local histogram feature approach has been proposed and shown to be effective. However, the effectiveness of a combined feature vector of local histogram and local slice feature vectors is not clear. In this paper, more effectively to classify diffuse lung opacities in HRCT images, we explore the combined feature vector of local histogram and local slice feature vectors. Furthermore, we examine the effects of normalization, rescaling and standardization, for the combined feature vector.
\end{abstract}

Index Terms -Diffuse lung opacities, combined feature vector, local histogram feature, local slice feature, HRCT images, CAD system.

\section{INTRODUCTION}

Thin-section computed tomography (HRCT) images are widely used for diagnosis of diffuse lung opacities [1]. The diffuse lung opacities classification in HRCT images is an important step for developing a computer-aided diagnosis (CAD) system [2], [3]. The HRCT images $(512 \times 512$ pixels, 12 bits / pixel, $0.35 \mathrm{~mm} /$ pixel) were obtained from 70 patients with a variety of diffuse lung diseases. In HRCT images, we selected 500 regions of interests (ROIs) with 4 classes of typical diffuse lung opacities (reticular, nodular, ground-glass, and consolidation) and one normal class. Fig. 1 shows ROI images with 4 classes of typical diffuse lung opacities and one normal class. In this paper, we use a $15 \times 15$ ROI size.

In our previous studies [4]-[6], each of a local histogram feature vector approach and a slice feature vector approach has been proposed and shown to be effective independently. A local histogram feature vector approach seems directory to reflect the CT values. On the other hand, a local slice feature vector approach may reflect shapes of the typical diffuse lung opacities. It is reported that combining different features leads to a better classification performance than that of individual features [7]. Therefore, we consider combining local

Manuscript received July 7, 2015; revised December 29, 2015. This work was supported by JSPS KAKENHI Grant Number 25330357.

Yoshihiro Mitani is with the National Institute of Technology, Ube College, Ube, Japan (e-mail: mitani@ ube-k.ac.jp).

Yusuke Fujita, Naofumi Matsunaga, and Yoshihiko Hamamoto are with Yamaguchi University, Ube, Japan. histogram and local slice feature vectors.

In this paper, in order to improve further the classification performance of the CAD system for classifying diffuse lung opacities in HRCT images, we explore the combination of local histogram and local slice feature vectors. Furthermore, we examine the effects of normalization, rescaling and standardization, for the combined feature vector. From the experimental result, the use of normalization techniques has been shown to be effective slightly.

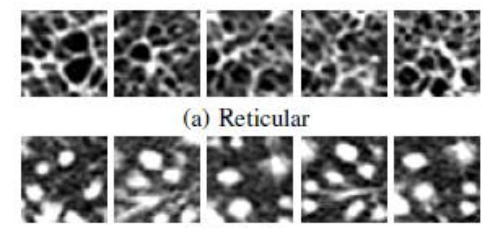

(b) Nodular

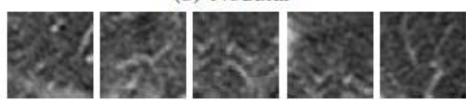

(c) Ground-glass

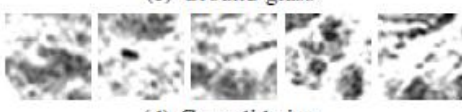

(d) Consolidation

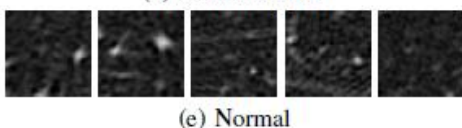

Fig. 1. ROI images with 4 classes of typical diffuse lung opacities and one normal class.

\section{PREVIOUS WORKS}

In the previous study [4]-[6], we have proposed a local histogram feature vector approach [4] and a local slice feature vector approach [5]. By focusing the local ranges of the CT values, we can get a rich feature to be effectively classified. These approaches have common characteristics. Thus, we set a window, which is determined by a window level and a window width, to the histogram. By setting a window successfully, a rich feature to be classified can be obtained. We showed each of a local histogram feature vector approach and a local slice feature vector approach.

\section{A. A Local Histogram Feature Vector Approach [4]}

A histogram, which consists of $\mathrm{x}$ - and $\mathrm{y}$ - axes, is obtained from the gray ROI image. The $\mathrm{x}-$ and $\mathrm{y}$ - axes show the CT value and its frequency, respectively. The histogram feature vector is obtained by arranging the frequency of a corresponding CT value from the histogram. The ROI image has 4,096 gray levels. The dimensionality of the histogram feature vector is 4,096 . Since the dimensionality is very large, 
we quantize the gray ROI image. In quantizing the gray ROI image, the threshold levels are equally divided.

In a local histogram feature vector approach, we make a local histogram by setting a window. Fig. 2 shows difference between conventional and local histogram feature vector approaches. A conventional histogram covers all range of CT values in general. On the other hand, a local histogram covers the only selected range of $\mathrm{CT}$ values. The range is decided by setting a window. The window is defined by a window level and a window width. Note that when the window level and window width are 2,048 and 4,096, the local histogram feature vector approach is equal with the conventional one. In the local histogram feature vector approach, we can select the number of bins in the local histogram. In Fig. 2(b), the bin size or dimensionality is 5 . For simplicity, the bin width is equally divided. All the bin widths are the same.

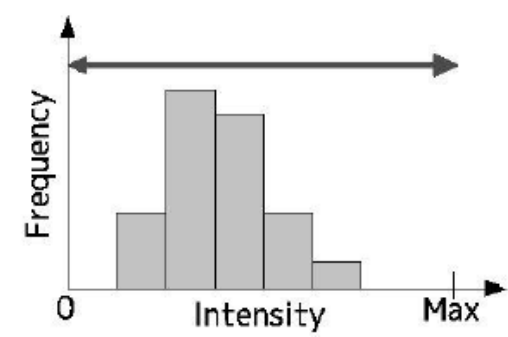

(a) A conventional histogram feature vector approach.

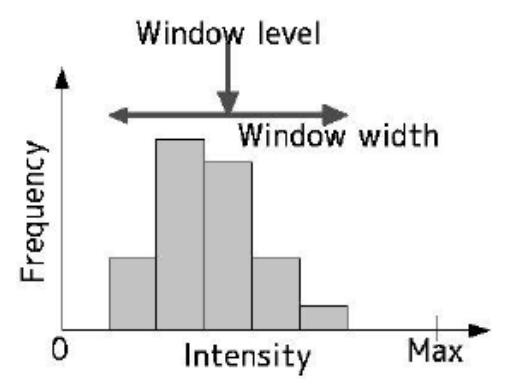

(b) A local histogram feature vector approach.

Fig. 2. Difference between conventional and local histogram feature vector approaches.

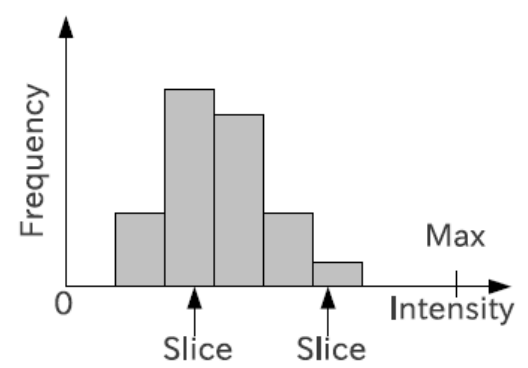

(a) A conventional slice feature vector approach.

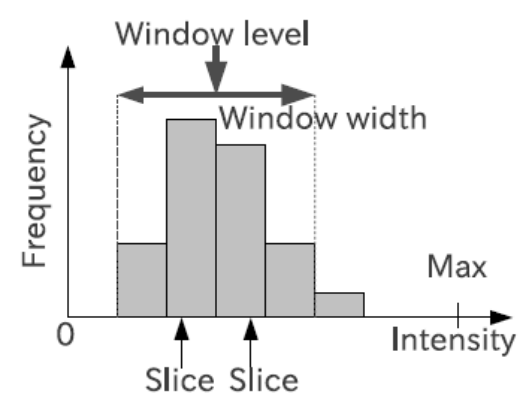

(b) A local slice feature vector approach.

Fig. 3. Difference between conventional and local slice feature vector approaches.

\section{B. A Local Slice Feature Vector Approach [5]}

A local slice feature vector approach uses a histogram like a local histogram feature vector approach. In a slice feature vector approach, the binary image to be sliced is used. The element of a slice feature vector is a ratio of white pixels in the binary image. Each of elements is produced by a binary image to be sliced by setting a threshold value. For simplicity, the slice width is equally divided. All the slice widths are the same.

The local slice feature vector is obtained based on a binary image to be sliced from a local histogram covered with a window. Fig. 3 shows difference between conventional and local slice feature vector approaches. A conventional slice feature vector approach [6] covers all range of CT values in general. On the other hand, a local slice feature vector approach covers the only selected range of CT values. The range is decided by setting a window, a window level and a window width, respectively. Note that when the window level and window width are 2,048 and 4,096, a local slice feature vector approach is equal with the conventional one. In the local slice feature vector approach, we can select the number of slices in the local histogram. In Fig. 3(b), the slice size or dimensionality is 2 . For simplicity, the slice width is equally divided. All the slice widths are the same. A detail procedure of a local slice feature vector approach has been shown in the literature [5].

\section{A PROPOSED METHOD}

In order to further improve the classification performance of the diffuse lung opacities in HRCT images, we explore the combination of local histogram and local slice feature vectors. In this paper, we make a combined feature vector as follows. Suppose that a local histogram feature vector $\boldsymbol{u}=\left[u_{1}, u_{2}, \ldots, u_{n}\right]^{T}$ and a local slice feature vector $\boldsymbol{v}=\left[v_{1}, v_{2}, \ldots, v_{m}\right]^{T}$ are given. The combined feature vector $\boldsymbol{w}$ is obtained by

$$
\boldsymbol{w}=\left[t u_{1}, t u_{2}, \ldots, t u_{n},(1-t) v_{1},(1-t) v_{2}, \ldots,(1-t) v_{m}\right]^{T},
$$

where $t(0<=t<=1)$ is a weight. The dimensionality of the combined feature vector is $n+m$. In general, the dimensionality of the combined feature vector becomes large. The combined feature vector is essentially equal to the local histogram feature vector when $t=1.0$, and to the local slice feature vector when $t=0.0$. When $t=0.5$, this means simply arranging the local histogram and local slice features. By appropriately adjusting a value of $t$, the improvement of the classification performance of the combined feature vector is expected.

Furthermore, we develop the combined feature vector with normalization. The normalization technique is applied to each of a feature vector. The combined feature vector is expected to be smoothed for gaps between a local histogram feature vector and a local slice feature vector. The normalization technique may not necessarily be improved. In this paper, we examine two types of normalization techniques, rescaling and standardization. We showed each of a rescaling technique and a standardization technique.

\section{A. A Rescaling Technique}

The method is rescaling the range of features to scale the 
range $[0,1]$. If the raw data $x$ is given, the rescale data $x^{\prime}$ is as follows.

$$
x^{\prime}=\left(x-x_{\min }\right) /\left(x_{\max }-x_{\min }\right) .
$$

Here, $x_{\max }$ and $x_{\min }$ are the maximum and minimum values of the raw data $x$, respectively. The range of the raw data $\left[x_{\min }\right.$, $\left.x_{\max }\right]$ becomes the range $[0,1]$.

\section{B. A Standardization Technique}

The method makes the values of each element in the data have zero-mean and unit variance. If the raw data $x$ is given, the standardization data $x^{\prime}$ is as follows.

$$
x^{\prime}=\left(x-x_{\text {ave }}\right) / x_{s d} .
$$

Here, $x_{a v e}$ and $x_{s d}$ are the mean and standard deviation of the raw data $x$, respectively. The raw data $x$ becomes the normal distribution $N(0,1)$.

\section{EXPERIMENTAL RESULTS}

The effectiveness of the proposed method is examined in terms of the error rate. The error rate $P_{\mathrm{e}}$ is defined as follows: $P_{\mathrm{e}}=100 \times$ No. of test samples misclassified / No. of all test samples [\%]

In order for the estimated error rate to be reliable in predicting the future classification performance of the CAD system, the training and test samples must be statistically independent [8]. In error rate estimation literature, the holdout method has been successfully used, because it maintains the statistical independence between the training and test samples [9]. In order to evaluate the proposed method, the average error rate and the $95 \%$ confidence interval were estimated.

- Step1 Divide 500 available samples into 250 training and 250 test samples at random

- Step2 Compute the error rate by using the local mean-based classifier [10]

- Step3 Repeat Steps 1 and 2, 100 times independently

- Step4 Calculate the average error rate and the $95 \%$ confidence interval.

The local mean-based classifier is known to be robust for outliers [10]. In the experiments, we used the local mean-based classifier with $r=5$.

The purpose of the experiment 1 is to show an individual performance of a local histogram feature vector approach and a local slice feature vector approach. Each of the dimensionality was 8 , and we used an 8-dimensional feature vector through all the experiments. We vary the window, a window level and a window width. Tables I and II are the results of the experiment 1 on the minimum average error rate. The minimum average error rate means the optimal window setting in terms of the average error rate. In the local histogram feature vector approach, the optimal window level is 500 and the optimal window width is 1,700 . On the other hand, in the local slice feature vector approach, the optimal window level is 700 and the optimal window width is 1,600. From both the results of the Tables I and II, localization by setting a window seems very effective. In the following experiments, we adopted these optimal window settings.

The purpose of the experiment 2 is to examine the combined feature vector of local histogram and local slice feature vectors. We made the combined feature vector and its dimensionality was $16(=8+8)$. We vary the values of $t$. The $t$ is a weight which controls a balance of a local histogram feature vector and a local slice feature vector. In the experiment, the values of $t$ were from 0.0 to 1.0. Again, when $t=0.0$, the combined feature vector is equal to a local slice feature vector. On the other hand, when $t=1.0$, the combined feature vector is equal to a local histogram feature vector. Table III shows the influence of values of $t$ in the combined feature vector of local histogram and local slice feature vectors. From the result of the Table III, there is little influence of values of $t$. When $t=0.0$, the combined feature vector performance gives the best. This means that only the local slice feature vector approach is effective. Unfortunately the effectiveness of the combined feature vector seems not clear. The range of an individual feature vector, a local histogram feature vector and a local slice feature vector, may differ largely.

TABLE I: COMPARISON OF THE CONVENTIONAL HISTOGRAM AND LOCAL HISTOGRAM FEATURE VECTOR APPROACHES

\begin{tabular}{|c|c|}
\hline Conventional histogram & Local histogram \\
\hline 16.86 & 4.34 \\
$16.43,17.17$ & $4.11,4.56$ \\
\hline
\end{tabular}

The upper is an average error rate (\%) and the lower is the $95 \%$ confidence interval.

TABLE II: COMPARISON OF THE CONVENTIONAL SLICE AND LOCAL SLICE FEATURE VECTOR APPROACHES

\begin{tabular}{|c|c|}
\hline Conventional slice & Local slice \\
\hline 11.48 & 4.01 \\
$11.16,11.79$ & $3.78,4.25$ \\
\hline
\end{tabular}

The upper is the average error rate (\%) and the lower is the $95 \%$ confidence interval.

TABLE III: INFLUENCE OF VALUES OF T IN THE COMBINED FEATURE VECTOR OF LOCAL HISTOGRAM AND LOCAL SLICE FEATURE VECTORS

\begin{tabular}{|l|l|l|l|l|l|}
\hline \multicolumn{6}{|l|}{ Values of $t$} \\
\hline 0.0 & 0.2 & 0.4 & 0.6 & 0.8 & 1.0 \\
\hline 4.01 & 4.01 & 4.01 & 4.01 & 4.02 & 4.34 \\
3.78, & 3.78, & 3.78, & 3.78, & 3.78, & $4.11,4.56$ \\
4.25 & 4.25 & 4.25 & 4.25 & 4.25 & \\
\hline
\end{tabular}

The upper is the average error rate (\%) and the lower is the $95 \%$ confidence interval.

TABLE IV: EFFECTS OF THE COMBINED FEATURE VECTOR WITH NORMALIZATION, RESCALING AND STANDARDIZATION

\begin{tabular}{|l|l|l|}
\hline $\begin{array}{l}\text { Conventional } \\
\text { method }\end{array}$ & $\begin{array}{l}\text { Rescaling } \\
\text { method }\end{array}$ & $\begin{array}{l}\text { Standardization } \\
\text { method }\end{array}$ \\
\hline 4.01 & 3.94 & 3.93 \\
$3.78,4.25$ & $3.70,4.18$ & $3.69,4.16$ \\
\hline
\end{tabular}

The upper is the average error rate (\%) and the lower is the $95 \%$ confidence inteval.

The purpose of the experiment 3 is to investigate the combined feature vector with normalization. By normalization of the data, we expected the combined feature vector to be smoothed for irregularities between a local histogram feature vector and a local slice feature vector. Note that the normalization technique is applied to not all of a combined feature vector but each of a feature vector, a local histogram feature vector and a local slice feature vector. In the experiment, we vary the values of $t$ and find the optimal value of $t$ in terms of the error rate. Table IV shows the effects of the combined feature vector with normalization, rescaling and standardization. All the results give the minimum average 
error rate with the optimal value of $t$. In the rescaling, the optimal value of $t$ is 0.3 . On the other hand, in the standardization, the optimal value of $t$ is 0.2 . From the result of the Table IV, both the normalization techniques, rescaling and standardization, improve the average error rate very slightly. However, from the viewpoint of the $95 \%$ confidence interval, the effect of the normalization techniques seems not clear.

\section{CONCLUSIONS}

In this paper, in order to improve further the classification performance of the CAD system for classifying diffuse lung opacities in HRCT images, we have explored the combination of local histogram and local slice feature vectors. Furthermore, we have examined the effects of normalization, rescaling and standardization, for the combined feature vector. From the experimental results, the effectiveness of the combined feature vector with the normalization techniques has been appeared slightly. In the future, we explore the feature selection for a combine feature vector.

\section{REFERENCES}

[1] I. Sluimer, A. Schilham, M. Prokop, and B. van Ginneken, "Computer analysis of computed tomography scans of the lung: A survey," IEEE Trans., Medical Imaging, vol. 25, no. 4, pp. 385-405, 2006.

[2] J. S. Duncan and N. Ayache, "Medical image analysis: Progress over two decades and the challenges ahead," IEEE Trans., PAMI-22, no. 1, pp. 85-106, 2000.

[3] K. Doi, "Computer-aided diagnosis in medical imaging: Historical review, current status and future potential," Computerized Medical Imaging and Graphics, no. 31, pp. 198-211, 2007.
[4] Y. Mitani, Y. Fujita, N. Matsunaga, and Y. Hamamoto, "The use of a local histogram feature vector for classifying diffuse lung opacities in high-resolution computed tomography," International Journal of Advancements in Computing Technology, vol. 4, no. 2, pp. 22-28, 2012.

[5] Y. Mitani, Y. Fujita, N. Matsunaga, and Y. Hamamoto, "A study of local slice features for a lung opacities classification in thin-section computed tomography images," in Proc. IASTED International Conference on Signal and Image Processing, 2013, pp. 460-463.

[6] Y. Mitani, Y. Fujita, N. Matsunaga, and Y. Hamamoto, "The use of a slice feature vector of classifying diffuse lung opacities in high-resolution computed tomography images," in Proc. 4th International Conference on Computational Intelligence, Modeling and Simulation, 2012, pp. 60-63.

[7] A. Jain and D. Zongker, "Feature selection: Evaluation, application, and small sample performance," IEEE Trans., PAMI-19, no. 2, pp. 153-158, 1997.

[8] P. A. Devijver and J. Kittler, Pattern Recognition: A Statistical Approach, Prentice Hall, 1982.

[9] K. Fukunaga, Introduction to Statistical Pattern Recognition, 2nd ed. Academic Press, 1990

[10] Y. Mitani and Y. Hamamoto, "A local mean-based nonparametric classifier," Pattern Recognition Letters, vol. 27, no. 10, pp. 1151-1159, 2006.

Yoshihiro Mitani got the $\mathrm{PhD}$ degree and is currently a professor at National Institute of Technology, Ube College, Japan. His research interest include pattern recognition and image processing techniques. $\mathrm{He}$ is a member of IEEE.

Yusuke Fujita got the $\mathrm{PhD}$ degree and is currently an assistant professor at Yamaguchi University, Japan. His research interests include pattern recognition and image processing techniques.

Yoshihiko Hamamoto got the $\mathrm{PhD}$ degree and is currently a professor at Yamaguchi University, Japan. His research interests include pattern recognition. He is a member of IEEE. 